\title{
Computed tomographic features for differentiating benign from malignant liver lesions in dogs
}

\author{
Rommaneeya LEELA-ARPORN ${ }^{1,2)}$, Hiroshi OHTA ${ }^{1)}$, Genya SHIMBO ${ }^{2,3)}$, \\ Kiwamu HANAZONO ${ }^{3,4)}$, Tatsuyuki OSUGA ${ }^{2,3)}$, Keitaro MORISHITA ${ }^{2,3)}$, \\ Noboru SASAKI ${ }^{1)}$ and Mitsuyoshi TAKIGUCHI ${ }^{1) *}$ \\ 1)Laboratory of Veterinary Internal Medicine, Department of Veterinary Clinical Sciences, \\ Graduate School of Veterinary Medicine, Hokkaido University, Sapporo, Hokkaido 060-0818, Japan \\ 2) Faculty of Veterinary Medicine and Applied Zoology, HRH Princess Chulabhorn College of Medical Science, \\ Chulabhorn Royal Academy, Lak Si, Bangkok 10210, Thailand \\ ${ }^{3)}$ Veterinary Teaching Hospital, Graduate School of Veterinary Medicine, Hokkaido University, Sapporo, \\ Hokkaido 060-0818, Japan \\ ${ }^{4)}$ School of Veterinary Medicine, Department of Veterinary Medicine, Rakuno Gakuen University, Ebetsu, \\ Hokkaido 069-8501, Japan
}

J. Vet. Med. Sci.

81(12): 1697-1704, 2019

doi: 10.1292/jvms.19-0278

Received: 20 May 2019

Accepted: 24 September 2019 Advanced Epub:

10 October 2019
ABSTRACT. Thus far, there are few computed tomography (CT) characteristics that can distinguish benign and malignant etiologies. The criteria are complex, subjective, and difficult to use in clinical applications due to the high level of experience needed. This study aimed to identify practical CT variables and their clinical relevance for broadly classifying histopathological diagnoses as benign or malignant. In this prospective study, all dogs with liver nodules or masses that underwent CT examination and subsequent histopathological diagnosis were included. Signalments, CT findings and histopathological diagnoses were recorded. Seventy liver nodules or masses in 57 dogs were diagnosed, comprising 18 benign and 52 malignant lesions. Twenty-three qualitative and quantitative $\mathrm{CT}$ variables were evaluated using univariate and stepwise multivariate analyses, respectively. Two variables, namely, the postcontrast enhancement pattern of the lesion in the delayed phase (heterogeneous; odds ratio (OR): 14.7, 95\% confidence interval (Cl): 0.82-262.03, $P=0.0429$ ) and the maximal transverse diameter of the lesion ( $>4.5 \mathrm{~cm}$; OR: $33.3,95 \% \mathrm{Cl}: 2.29-$ $484.18, P=0.0006$ ), were significantly related to the differentiation of benign from malignant liver lesions, with an area under the curve of 0.8910 , representing an accuracy of $88.6 \%$. These findings indicate that features from triple-phase $\mathrm{CT}$ can provide information for distinguishing pathological varieties of focal liver lesions and for clinical decision making. Evaluations of the maximal transverse diameter and postcontrast enhancement pattern of the lesion included simple CT features for predicting liver malignancy with high accuracy in clinical settings.

KEY WORDS: canine, classification, computed tomography, liver, neoplasia

Focal liver lesions that present as nodules or masses in dogs may be relatively common findings. Relevant clinical signs would be a reason for visiting an animal hospital. The lesion could reflect pathological varieties, including benign or malignant conditions, and appropriate management of the lesion depends on determining the diagnosis of the pathology. As the prognosis and specific treatment of each type of lesion can vary, it is important to tentatively diagnose the lesion to obtain information regarding the biological behavior of the lesion prior to treatment planning. However, liver biopsy, which is the gold standard for a definitive diagnosis of lesion type [14], is invasive and can cause life-threatening complications [2, 11]. Therefore, noninvasive diagnostic imaging could serve as a valuable method to distinguish benign liver lesions from malignant ones, although determining the nature of a nodule or mass via imaging diagnosis remains challenging.

Recently, technological advancements in computed tomography (CT) systems have improved the image quality and enabled quick scanning, which helps reduce the radiation dose and the need for prolonged sedation or anesthesia [3, 5]. Consequently, the number of dogs undergoing abdominal CT examination has increased, and increasing numbers of nodules or masses have been identified as incidental findings. In humans, CT has been used to examine various pathological hepatic conditions via an enhancement pattern following intravenous administration of contrast medium $[1,7,8,16,17]$. In dogs, previous studies have 
revealed that triple-phase helical CT characteristics are useful for differentiating among hepatocellular carcinoma, hepatocellular adenoma, nodular hyperplasia and metastasis $[4,10,18]$ since dynamic CT can reveal differences in vascularization that are of diagnostic significance $[8,12,15]$. However, its diagnostic performance in differentiating benign from malignant liver lesions has not been sufficiently clarified due to the complexity of using contrast uptake characteristics [9, 15], which is a subjective evaluation that can vary among both veterinary radiologists and clinicians. A previous study reported that the lowest delayed phase absolute enhancement of a mass is able to distinguish benign from malignant liver masses with high accuracy [5]. However, this subjective criterion has been precluded from application in clinical settings, as considerable clinical experience is needed. To the best of our knowledge, CT characteristics remain challenging for differentiating benign from malignant liver lesions, although a few studies have investigated the association between CT features and pathological varieties of liver lesions [5, 9], and determining applicable CT features for predicting liver malignancy in clinical practice could aid in clinical decision making for treatment.

Therefore, the purpose of this study was to determine the diagnostic value of dynamic CT images in order to identify CT variables that could be useful for classifying benign and malignant liver lesions in clinical practice.

\section{MATERIALS AND METHODS}

\section{Study design and study population}

This study was a prospective study conducted in accordance with the Hokkaido University Animal Care and Use Committee. Medical records of the dogs attending the Hokkaido University Veterinary Teaching Hospital were searched from April 2016 to June 2019 for all dogs with liver nodules or masses. Consent was obtained from the owners of all dogs recruited into this study.

The inclusion criteria of this study were dogs with liver nodules or masses that underwent abdominal CT examination before obtaining surgical resection of the lesion. All histopathological diagnoses were performed by a single board-certified pathologist.

Dogs were excluded from the study if they did not undergo CT examination or did not have a definitive diagnosis of a lesion that was histopathologically confirmed.

\section{CT procedures}

All CT examinations were performed using an 80-row multidetector CT scanner (Aquilion PRIME, Toshiba Medical Systems, Tochigi, Japan) by an experienced radiologist (KH or GS). Helical CT scans that included precontrast and postcontrast images in the arterial, portal and delayed phases through the liver were routinely performed for all abdominal CT scans. Anesthesia for all dogs was induced by propofol, and the dogs were positioned in dorsal or sternal recumbency. General anesthesia was maintained by isoflurane inhalation through CT examination. The scan settings included a slice thickness of $3 \mathrm{~mm}$, helical pitch of 0.813 , tube rotation time of $0.5 \mathrm{sec}$, X-ray tube potential of $120 \mathrm{kVp}$ and X-ray tube current of 60-500 mA, which were automatically calculated by a commercial software package (Sure Exposure 3D; Toshiba Medical Systems, Tochigi, Japan). All helical scans were initiated at the diaphragmatic dome and extended caudally to the level of the pelvic inlet. Following precontrast CT scanning, iohexol (Omnipaque 300, GE Healthcare, Oslo, Norway) was used as a contrast medium and was administered at a dose of $2 \mathrm{ml} / \mathrm{kg}$ $(600 \mathrm{mg}$ iodine $/ \mathrm{kg})$ via the cephalic vein with a power injector over an injection duration of 20-30 sec. Postcontrast images were obtained using the bolus tracking technique. The trigger threshold for the arterial scan was set at 200 Hounsfield units (HU) of the abdominal aorta. After triggering for $20 \mathrm{sec}$, the arterial phase scan was automatically initiated. The portal phase was initiated at $20 \mathrm{sec}$ after the end of the arterial phase scan. Three min after the beginning of the injection of contrast medium, a delayed phase scan was obtained.

\section{Data collection and diagnostic criteria}

Medical histories of the dogs included in the study were reviewed, and the following data were extracted: signalment, including age, sex, breed and body weight, and histopathological diagnosis of the liver lesion.

CT images of all included dogs were reviewed using DICOM viewing software (OsiriX, Pixmeo SARL, Bernex, Switzerland) by a doctoral student (RL) trained in internal medicine and diagnostic imaging that was unaware of the final diagnosis at the time of image review. The number of liver nodules or masses in each dog was recorded during CT image evaluation, which included both qualitative and quantitative variables.

The following qualitive variables were recorded: location; appearance of the lesion margin (well-defined or ill-defined); surface appearance (lobulated or smooth); capsule formation, which was defined as a thin or thick band that partially or completely surrounded the tumor at different attenuations of postcontrast images (presence or absence); and blood vessel distribution within each lesion during arterial enhancement (central, peripheral or diffuse). Central enhancement was defined as the presence of blood vessels in the central area of the lesion. Peripheral and diffuse enhancements were defined as the presence of blood vessels surrounding the marginal area of the lesion and blood vessels extending across the entire lesion, respectively. Parenchymal homogeneity of the lesion in precontrast images and the postcontrast enhancement pattern of the lesion in postcontrast images were defined as the uniformity of lesion enhancement (homogenous or heterogeneous) by subjective evaluation. The overall attenuation of the lesion relative to normal liver parenchyma in postcontrast images (hypo-, iso- or hyperattenuation) was also recorded by measuring the contrast values in HU. Attenuation of the lesion, excluding calcification, vessels and nonenhanced regions that might be a cyst, necrosis or hemorrhage, and surrounding liver parenchyma was measured using a circular region of interest (ROI) of approximately $30 \mathrm{~mm}^{2}$ within the 3 areas of interest for each phase. The classification of attenuation of the lesion was defined by a cutoff of $10 \mathrm{HU}$ as described previously [10]. Hyperattenuation was defined as a lesion contrast value at least $10 \mathrm{HU}$ greater than 
that of the liver parenchyma. Isoattenuation was defined as a lesion contrast value that was no greater or less than 10 HU compared to that of the liver parenchyma. Hypoattenuation was defined as a lesion contrast value of at least $10 \mathrm{HU}$ less than that of the liver parenchyma.

The following quantitative variables were recorded: maximal transverse diameter of the lesion (cm), mean attenuation of normal liver parenchyma in each phase (HU), mean attenuation of the lesion in each phase (HU), relative attenuation of the lesion in each phase (mean attenuation of the lesion-mean attenuation of normal liver parenchyma) and the volume of the lesion $\left(\mathrm{cm}^{3}\right)$ by generating volume-rendered images of the lesion. Volume-rendered images were carefully constructed by placing an automated closed polygon ROI on the entire nodule or mass in transverse slices from the most cranial to the most caudal part of the lesion. The volume of the lesion was automatically computed from the sum of the volumes of all the ROIs of all the slices.

All medical records and CT data of all included dogs were compiled by one observer (RL).

\section{Statistical analysis}

Statistical analysis was performed using commercial software (JMP Pro, version 14.0.0; SAS Institute Inc., Cary, NC, U.S.A.) by one investigator (RL). The pathological conditions from histopathological diagnosis of the lesion were simply classified as benign or malignant lesions for comparisons of CT variables between both conditions using univariate and multivariate analyses. The normality of continuous data, including age, body weight, maximal transverse diameter of the lesion, volume of the lesion, attenuation of the lesion and normal liver parenchyma and relative attenuation of the lesion, was assessed by the Shapiro-Wilk test.

For the univariate analysis, continuous variables were analyzed using Student's $t$-test and the Mann-Whitney $U$ test for normally distributed and nonnormally distributed data, respectively, and are presented as the mean \pm standard deviation. Categorical variables, including sex, breed, location, appearance of the lesion margin, surfaces, capsule formation, blood vessel distribution, parenchymal homogeneity of the lesion and overall attenuation of the lesion, were assessed using Fisher's exact test or $\chi^{2}$ test and are presented as numbers and percentages.

For the multivariate analysis, a stepwise regression analysis was used to select the significant variables from the univariate analysis via forward selection with a $P$ value threshold $(P<0.15$ for inclusion and $P>0.2$ for exclusion) to identify independent variables that can classify benign and malignant liver lesions. The odds ratio (OR) and $95 \%$ confidence interval (CI) of each variable that was included in the multivariate model were calculated. Receiver operating characteristic curve analysis was performed to determine the diagnostic accuracy of the multivariate model and each independent variable. For all statistical analyses, a $P$ value $<0.05$ was considered statistically significant.

\section{RESULTS}

Fifty-seven dogs with 70 nodules or masses met the inclusion criteria and were enrolled in this study. The dogs included in this study consisted of 25 females (20 spayed) and 32 males (24 neutered). The average age at diagnosis was $11.2 \pm 2.4$ years. The average body weight of the dogs was $9.2 \pm 6.6 \mathrm{~kg}$. The dog breeds included 7 Chihuahuas, 4 Yorkshire Terriers, 4 Miniature Dachshunds, 4 Miniature Schnauzers, 4 Shiba Inus, 4 Shih Tzus, 4 Welsh Corgis, 3 Mongrels, 3 Toy Poodles, 2 Beagles, 2 Border Collies, 2 Golden Retrievers, 2 Malteses, 2 Papillons, and one of each of the following breeds: Australian Shepherd, Boston Terrier, Brussels Griffon, Cairn Terrier, Jack Russel, Lasa Apso, Miniature Pinscher, Pekingese, Saluki and Shetland Sheepdog.

The histological findings revealed 52 malignant and 18 benign varieties of liver lesions. For malignant lesions, 43 were hepatocellular carcinomas, 4 were hepatocholangiocarcinomas, 2 were cholangiocellular carcinomas, 2 were undifferentiated sarcomas, and 1 was a hemangiosarcoma. Benign lesions included 14 nodular hyperplasias, 1 cholangiocellular adenoma, 1 glycogen accumulation, 1 hematoma and 1 portal vein hypoplasia.

The evaluation of $23 \mathrm{CT}$ variables, including 12 qualitative and 11 quantitative variables, was included in the univariate analysis. Among these variables, 7 qualitative variables, including the appearance of the lesion margin, surfaces, capsule formation, parenchymal homogeneity of the lesion in the precontrast image and postcontrast enhancement pattern of the lesion in postcontrast images, and 3 quantitative variables, including maximal transverse diameter, volume of the lesion and mean attenuation of liver parenchyma in the portal phase, were significantly different between the benign and malignant liver lesions (Tables 1 and 2 ).

All significant CT variables from the univariate analysis were included in the stepwise analysis. Among these variables, the maximal transverse diameter, appearance of the lesion margin, parenchymal homogeneity of the lesion in the precontrast image and postcontrast enhancement pattern of the lesion in the arterial and delayed phases were selected as candidate variables for multivariate analysis, as shown in Table 3. However, the multivariate analysis revealed that only the maximal transverse diameter $(P=0.0006)$ and postcontrast enhancement pattern of the lesion in the delayed phase $(P=0.0429)$ were found to be significant independent $\mathrm{CT}$ variables for the differentiation of benign from malignant focal liver lesions (Figs. 1 and 2).

Regarding these independent CT variables, the best cutoff value of the maximal transverse diameter for predicting liver malignancy was $>4.5 \mathrm{~cm}$ (OR: 33.3, 95\% CI: 2.29-484.18) based on the highest Youden's index according to receiver operating characteristic curve analysis. In addition, malignant pathological conditions were associated with the heterogeneous appearance of lesions in the delayed phase (OR: 14.7, 95\% CI: 0.82-262.03) (Table 3). The diagnostic performance of each variable and the combination of both variables for predicting liver malignancy is shown in Table 4. The results indicated that the combination of both independent variables had a higher predictive ability than each individual variable in predicting liver malignancy, with an area under the curve of 0.8910 . The performance measurement based on the combination of both independent variables presented an accuracy of $88.6 \%$, a sensitivity of $92.3 \%$ and a specificity of $77.8 \%$. 
Table 1. Qualitative characteristics of benign and malignant focal liver lesions

\begin{tabular}{|c|c|c|c|}
\hline Variables & Benign $(\mathrm{n}=18)$ & Malignant $(\mathrm{n}=52)$ & $P$ value \\
\hline Location, n (\%) & & & 0.2384 \\
\hline Left lateral lobe & $7(38.9)$ & $15(28.9)$ & \\
\hline Left medial lobe & $1(5.6)$ & $7(13.5)$ & \\
\hline Right lateral lobe & $4(22.2)$ & $10(19.2)$ & \\
\hline Right medial lobe & $1(5.6)$ & $6(11.5)$ & \\
\hline Quadrate lobe & $3(16.7)$ & $1(1.9)$ & \\
\hline Caudate process of the caudate lobe & $1(5.6)$ & $7(13.5)$ & \\
\hline Papillary process of the caudate lobe & $1(5.6)$ & $6(11.5)$ & \\
\hline Surfaces, n (\%) & & & $0.0163^{\mathrm{a})}$ \\
\hline Lobulated & $1(5.6)$ & $18(34.6)$ & \\
\hline Smooth & $17(94.4)$ & $34(65.4)$ & \\
\hline Appearance of the lesion margin, $n(\%)$ & & & $0.0019^{\mathrm{a})}$ \\
\hline Well-defined & $11(61.1)$ & $49(94.2)$ & \\
\hline Ill-defined & $7(38.9)$ & $3(5.8)$ & \\
\hline Capsule formation, $\mathrm{n}(\%)$ & & & $0.0207^{\mathrm{a})}$ \\
\hline Presence & $2(11.1)$ & $22(42.3)$ & \\
\hline Absence & $16(88.9)$ & $31(57.7)$ & \\
\hline Blood vessel distribution, n (\%) & & & 0.0589 \\
\hline Central & $2(11.1)$ & $17(32.7)$ & \\
\hline Peripheral & $6(33.3)$ & $21(40.4)$ & \\
\hline Diffuse & $10(55.6)$ & $14(26.9)$ & \\
\hline Parenchymal homogeneity of the lesion in the precontrast image, $\mathrm{n}(\%)$ & & & $<0.0001^{\text {a) }}$ \\
\hline Homogenous & $17(94.4)$ & $16(30.8)$ & \\
\hline Heterogeneous & $1(5.6)$ & $36(69.2)$ & \\
\hline Postcontrast enhancement pattern of the lesion in the arterial phase, $\mathrm{n}(\%)$ & & & $0.0147^{\mathrm{a})}$ \\
\hline Homogenous & $10(55.6)$ & $11(21.2)$ & \\
\hline Heterogeneous & $8(44.4)$ & $41(78.9)$ & \\
\hline Postcontrast enhancement pattern of the lesion in the portal phase, $\mathrm{n}(\%)$ & & & $0.0004^{\mathrm{a})}$ \\
\hline Homogenous & $13(72.2)$ & $12(23.1)$ & \\
\hline Heterogeneous & $5(27.8)$ & $40(76.9)$ & \\
\hline Postcontrast enhancement pattern of the lesion in the delayed phase, $\mathrm{n}(\%)$ & & & $<0.0001^{\text {a) }}$ \\
\hline Homogenous & $15(83.3)$ & $13(25)$ & \\
\hline Heterogeneous & $3(16.7)$ & $39(75)$ & \\
\hline Overall attenuation in the arterial phase, $\mathrm{n}(\%)$ & & & 0.6328 \\
\hline Hyperattenuation & $7(38.9)$ & $14(26.9)$ & \\
\hline Isoattenuation & $3(16.7)$ & $10(19.2)$ & \\
\hline Hypoattenuation & $8(44.4)$ & $28(53.9)$ & \\
\hline Overall attenuation in the portal phase, $\mathrm{n}(\%)$ & & & 0.5201 \\
\hline Hyperattenuation & $3(16.7)$ & $5(9.6)$ & \\
\hline Isoattenuation & $4(22.2)$ & $8(15.4)$ & \\
\hline Hypoattenuation & $11(61.1)$ & $39(75)$ & \\
\hline Overall attenuation in the delayed phase, $\mathrm{n}(\%)$ & & & 0.0669 \\
\hline Hyperattenuation & $3(16.7)$ & $2(3.9)$ & \\
\hline Isoattenuation & $8(44.4)$ & $16(30.8)$ & \\
\hline Hypoattenuation & $7(38.9)$ & $34(65.4)$ & \\
\hline
\end{tabular}

a) $P$ values $<0.05$ were statistically significant.

\section{DISCUSSION}

In the present study, the maximal transverse diameter and postcontrast enhancement pattern of the lesion in the delayed phase were significantly related to the differentiation of pathological varieties of focal liver lesions, with an accuracy of $88.6 \%$ observed for triple-phase CT.

The maximal transverse diameter and postcontrast enhancement pattern of the lesion in the delayed phase, which were independent variables in the multivariate analysis, are considered suitable criteria for easily differentiating between benign and malignant liver lesions, although both were subjective variables. Using $4.5 \mathrm{~cm}$ as an optimal cutoff for the lesion, liver malignancy was independently predicted, with an accuracy of $77.1 \%$. This cutoff value for the maximal transverse diameter is consistent with that reported in previous studies on the ultrasonographic appearance of focal liver lesions [6, 13]. Additionally, parenchymal homogeneity of the lesion in the delayed phase independently classified liver lesions with an accuracy of $77.1 \%$, and a malignant 
Table 2. Quantitative characteristics of benign and malignant focal liver lesions

\begin{tabular}{|c|c|c|c|}
\hline Variables & Benign $(n=18)$ & Malignant $(\mathrm{n}=52)$ & $P$ value \\
\hline Maximal transverse diameter $(\mathrm{cm})$ & $3.1 \pm 1.4$ & $6.6 \pm 3.1$ & $<0.0001^{\mathrm{a})}$ \\
\hline Volume of the lesion $\left(\mathrm{cm}^{3}\right)$ & $21.3 \pm 24.5$ & $195.0 \pm 228.6$ & $<0.0001^{\text {a) }}$ \\
\hline \multicolumn{4}{|c|}{ Mean attenuation of liver parenchyma (HU) } \\
\hline Arterial phase & $121.2 \pm 16.1$ & $117.2 \pm 18.4$ & 0.3871 \\
\hline Portal phase & $172.1 \pm 26.3$ & $156 \pm 25.5$ & $0.0320^{\mathrm{a})}$ \\
\hline Delayed phase & $131.1 \pm 15.4$ & $125.3 \pm 16.8$ & 0.1879 \\
\hline \multicolumn{4}{|l|}{ Mean attenuation of the lesion (HU) } \\
\hline Arterial phase & $134.7 \pm 72.3$ & $112.0 \pm 49.6$ & 0.1524 \\
\hline Portal phase & $148.4 \pm 61.1$ & $118.4 \pm 44.9$ & 0.0683 \\
\hline Delayed phase & $119.7 \pm 37.5$ & $105.2 \pm 28.1$ & 0.1474 \\
\hline \multicolumn{4}{|l|}{ Relative attenuation of the lesion (HU) } \\
\hline Arterial phase & $13.5 \pm 71.9$ & $-5.2 \pm 52.5$ & 0.2915 \\
\hline Portal phase & $-23.7 \pm 53.9$ & $-37.6 \pm 41.7$ & 0.3283 \\
\hline Delayed phase & $-11.5 \pm 28.0$ & $-20.1 \pm 25.0$ & 0.1272 \\
\hline
\end{tabular}

a) $P$ values $<0.05$ were statistically significant; values are presented as the mean \pm standard deviation; HU, hounsfield units.

Table 3. Multivariate analysis to identify independent variables for differentiating malignant from benign focal liver lesions

\begin{tabular}{lccc}
\hline \multicolumn{1}{c}{ Variables } & Odds ratio & $95 \%$ CI & $P$ value \\
\hline $\begin{array}{l}\text { Maximal transverse diameter } \\
\quad>4.5 \mathrm{~cm}\end{array}$ & 33.32 & $2.29-484.2$ & $0.0006^{\mathrm{a})}$ \\
$\begin{array}{l}\text { Appearance of the lesion margin } \\
\quad \text { Well-defined }\end{array}$ & 8.04 & $0.71-91.5$ & 0.0559 \\
$\begin{array}{l}\text { Parenchymal homogeneity of the lesion in the precontrast image } \\
\quad \text { Heterogeneous }\end{array}$ & 4.30 & $0.36-51.6$ & 0.2213 \\
$\begin{array}{l}\text { Postcontrast enhancement pattern of the lesion in the arterial phase } \\
\quad \text { Heterogeneous }\end{array}$ & 0.25 & $0.02-3.28$ & 0.2554 \\
$\begin{array}{l}\text { Postcontrast enhancement pattern of the lesion in the delayed phase } \\
\text { Heterogeneous }\end{array}$ & 14.66 & $0.82-262.03$ & $0.0429^{\mathrm{a})}$ \\
\hline
\end{tabular}

a) $P$ values $<0.05$ were statistically significant; $\mathrm{CI}$, confidence interval.

variety of focal liver lesions presented with a significantly heterogeneous appearance in the delayed phase. This heterogeneous appearance could be related to the presence of a necrotic region within the mass, which is consistent with previous studies of CT characteristics of liver lesions $[4,5,10,18]$, revealing not only that the lowest absolute enhancement of the mass in the delayed phase of less than $37 \mathrm{HU}$ could predict malignant liver lesions and theoretically reflect the region of necrosis [5] but also that cystlike necrotic lesions tend to be found in hepatocellular carcinoma [4]. Regarding the results, both independent variables may reflect the aggressive biological behavior of liver malignancy, which could be indicated by a large lesion size and the presence of necrosis within a mass via the heterogeneous appearance of the lesion.

In this study, significant differences in the appearance of the lesion margin, parenchymal homogeneity of the lesion in the precontrast image and postcontrast enhancement pattern of the lesion in the arterial phase between benign and malignant focal liver lesions were observed in the univariate analysis; however, they may have fallen out of the multivariate analysis because they were less important than other factors. In addition, the present study did not find a significant difference in the blood vessel distribution in the lesions, and thus, this factor could not be used to distinguish benign from malignant liver lesions, which is consistent with the results of a previous study in dogs postulating that histopathological and vascular characteristics of liver neoplasms may vary between humans and dogs [5].

In this study, quantitative variables, including multiple attenuation values within a defined region for identifying the enhancement ability of the lesions after the uptake of contrast medium, were not measured due to the complexity of subjective evaluation and the experience of the observer. Furthermore, this study aimed to find practical variables that clinicians and veterinary radiologists can use to easily distinguish benign from malignant liver lesions in clinical practice. The measurement of multiple attenuation values may vary among observers due to the degree of subjectivity in placing the ROI on a defined region.

The high accuracy of diagnostic performance in differentiating benign from malignant focal liver lesions suggests that triplephase CT has the ability to identify lesions by their blood supply and contrast uptake characteristics during 3 distinct hepatic circulatory phases: the arterial, portal and delayed phases [8]. During the delayed phase, enhancement of the liver parenchyma is maximized, which may result in the clear evaluation of the postcontrast enhancement pattern of the lesion, as described in the present study.

One limitation of this study is its small sample size, with only 57 dogs enrolled. This sample size may affect the results and 

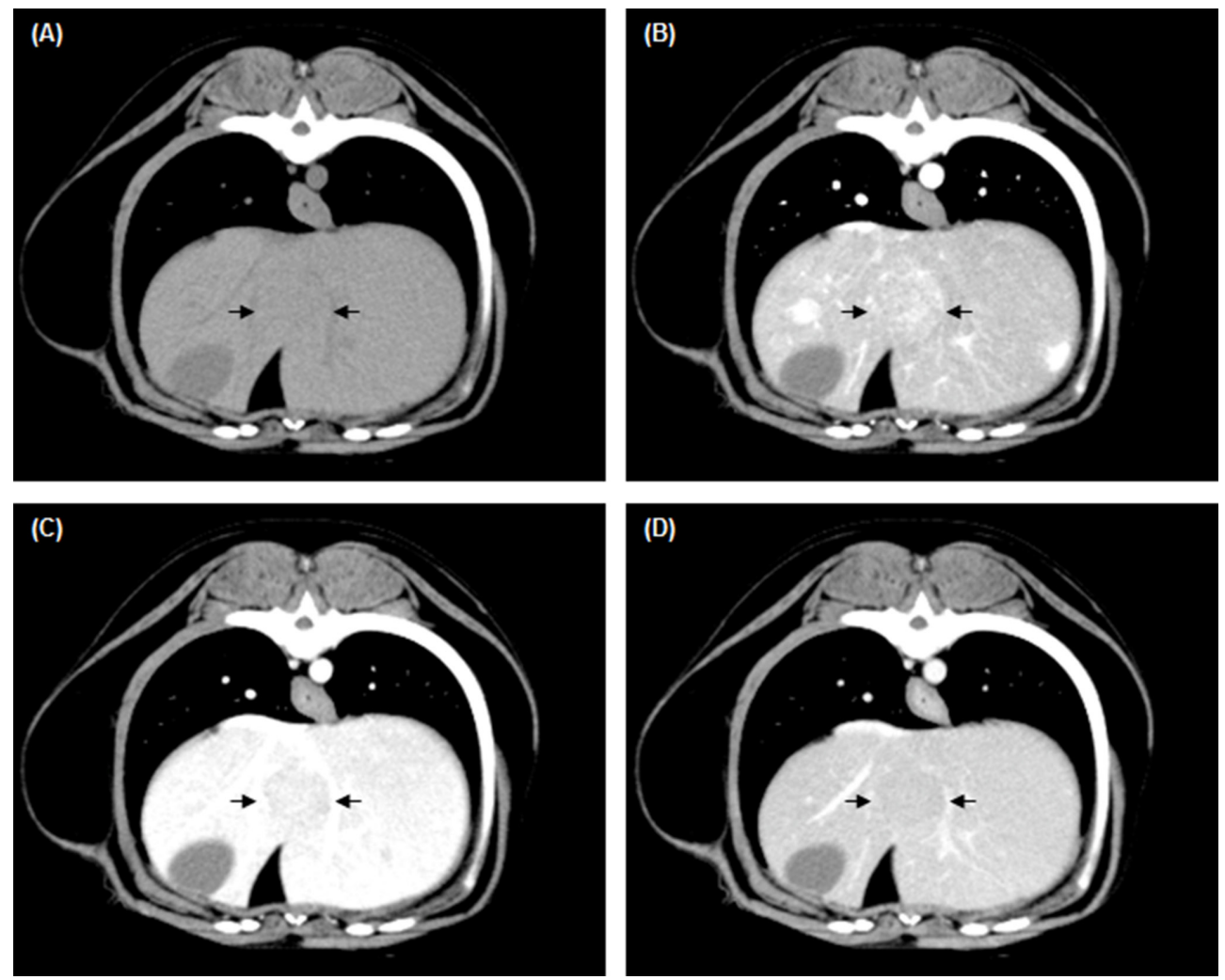

Fig. 1. Transverse computed tomography (CT) images of a dog with nodular hyperplasia. (A) A precontrast image presenting a 2-cm homogenous isoattenuating nodule (arrows) in the quadrate lobe. (B) A heterogeneous hyperattenuating nodule with diffuse enhancement (arrows) in the arterial phase. Homogenous isoattenuation of the nodule (arrows) in the (C) portal and (D) delayed phases.
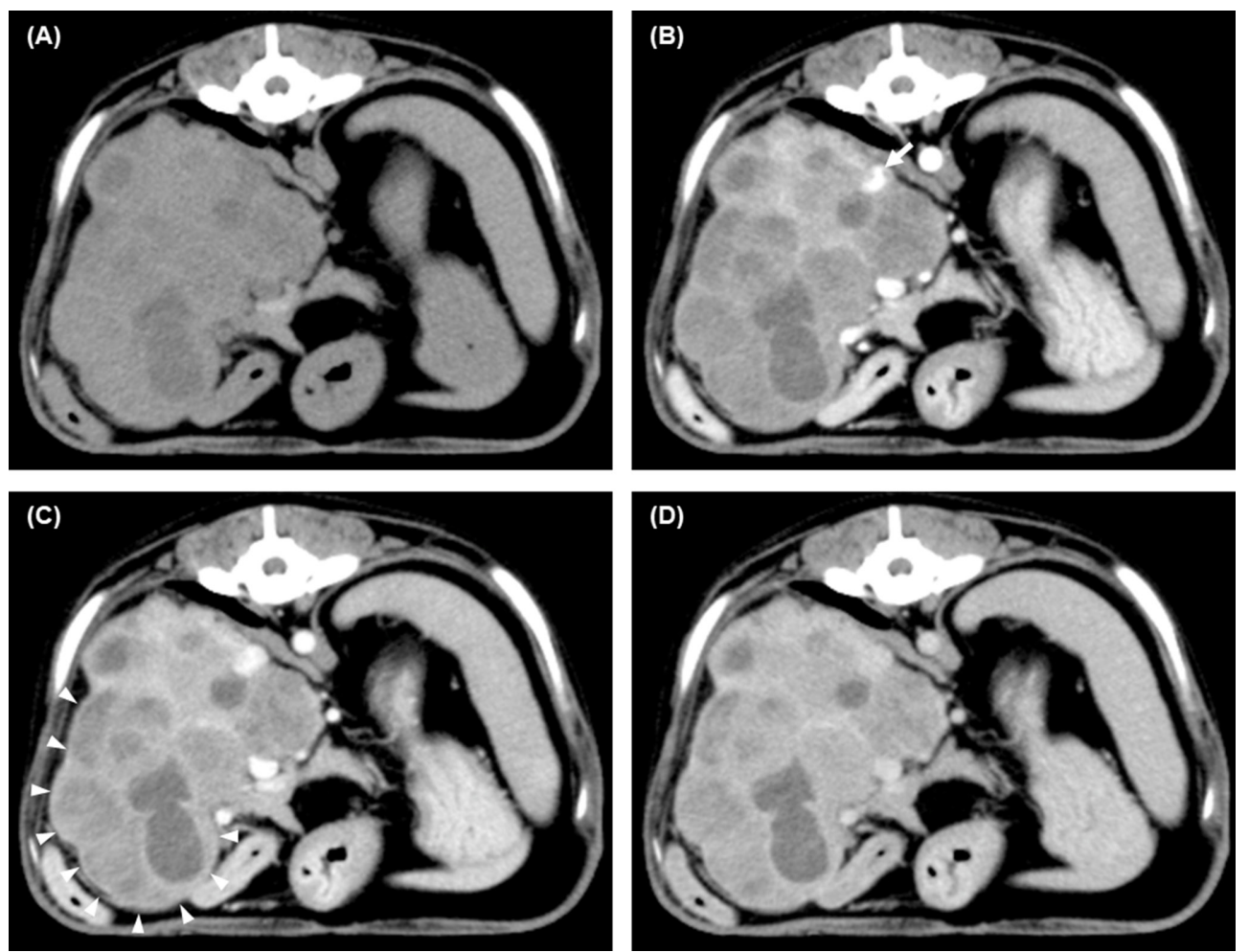

Fig. 2. Transverse computed tomography (CT) images of a dog with hepatocellular carcinoma. (A) A precontrast image presenting a 9-cm heterogeneous isoattenuating mass in the right lateral lobe. (B) Heterogeneous hypoattenuation of the mass in the arterial phase with arterial enhancement in the peripheral area of the lesion (arrow). (C) A thin band surrounding the heterogeneous hypoattenuating mass with different attenuations representing the tumor capsule (arrowheads) in the portal phase. (D) Heterogeneous isoattenuation of the mass in the delayed phase. 
Table 4. Diagnostic performance of independent computed tomography (CT) variables for predicting the malignancy of focal liver lesions

\begin{tabular}{lccc}
\hline \multicolumn{1}{c}{ Factors } & Both independent variables & $\begin{array}{c}\text { Postcontrast enhancement pattern of } \\
\text { the lesion in the delayed phase }\end{array}$ & Maximal transverse diameter \\
\hline Area under the curve & 0.8910 & 0.7917 & 0.8098 \\
Sensitivity (\%) & 92.3 & 75.0 & 73.1 \\
Specificity (\%) & 77.8 & 83.3 & 88.9 \\
Positive predictive value (\%) & 92.3 & 92.9 & 95.0 \\
Negative predictive value (\%) & 77.8 & 53.6 & 53.3 \\
Accuracy (\%) & 88.6 & 77.1 & 77.1 \\
\hline
\end{tabular}

accuracy of the CT variables used to distinguish benign from malignant focal liver lesions. Another limitation is the relative lack of variety in lesion types from the histopathologic results that were included in this study, likely leading to a number bias. It is possible that benign-looking malignancies were not taken to surgery and thus were also not included in this study. Therefore, our study population based on histologic examination could have affected the results of this study. Next, the discrepancies in the CT protocols used among studies in the literature may also affect the CT interpretation in each study. Moreover, the effect of the duration of contrast material injection on the arterial phase for the detection and classification of pathological varieties of focal liver lesions in veterinary medicine remains unclear. Therefore, another prospective study is warranted to investigate the effect of contrast material injection duration on arterial enhancement. Finally, CT measurement variables for interpretation vary between studies, leading to differences in the results due to subjectivity and observer variation. Despite these limitations, this study identified CT variables that can be used to easily differentiate benign from malignant lesions in the clinic using fixed criteria for each variable to minimize interobserver variability.

In conclusion, based on the small number of dogs with limited lesion types in this study, we found that CT variables, including the maximal transverse diameter and postcontrast enhancement pattern of the lesion in the delayed phase are independent variables that can distinguish benign and malignant liver lesions. The clinical relevance of both variables for predicting liver malignancy are high accuracy, supporting their clinical use. Although CT diagnosis cannot provide a specific diagnosis, which requires histopathological examination, the results of this study indicate that triple-phase CT can aid in the prediction of pathological varieties of focal liver lesions and can assist clinicians in clinical decision making. Further investigations with larger numbers of dogs presenting different types of malignant liver tumors and nonmalignant lesions should be conducted to support the results of this study and to provide a more in-depth investigation into CT characteristics of focal liver lesions within each specific classification due to heterogeneous prognoses among specific lesion diagnoses.

CONFLICT OF INTEREST. No third-party funding or support was received in connection with this study or the writing or publication of the manuscript. The authors declare that there are no conflicts of interest.

ACKNOWLEDGMENT. The authors thank Dr. Yumiko Kagawa, an American College of Veterinary Pathologists board-certified pathologist, for her help with the interpretation of all the histopathological findings.

\section{REFERENCES}

1. Akai, H., Kiryu, S., Matsuda, I., Satou, J., Takao, H., Tajima, T., Watanabe, Y., Imamura, H., Kokudo, N., Akahane, M. and Ohtomo, K. 2011. Detection of hepatocellular carcinoma by Gd-EOB-DTPA-enhanced liver MRI: comparison with triple phase 64 detector row helical CT. Eur. J. Radiol. 80: 310-315. [Medline] [CrossRef]

2. Bigge, L. A., Brown, D. J. and Penninck, D. G. 2001. Correlation between coagulation profile findings and bleeding complications after ultrasoundguided biopsies: 434 cases (1993-1996). J. Am. Anim. Hosp. Assoc. 37: 228-233. [Medline] [CrossRef]

3. Fields, E. L., Robertson, I. D., Osborne, J. A. and Brown, J. C. Jr. 2012. Comparison of abdominal computed tomography and abdominal ultrasound in sedated dogs. Vet. Radiol. Ultrasound 53: 513-517. [Medline] [CrossRef]

4. Fukushima, K., Kanemoto, H., Ohno, K., Takahashi, M., Nakashima, K., Fujino, Y., Uchida, K., Fujiwara, R., Nishimura, R. and Tsujimoto, H. 2012. CT characteristics of primary hepatic mass lesions in dogs. Vet. Radiol. Ultrasound 53: 252-257. [Medline]

5. Griebie, E. R., David, F. H., Ober, C. P., Feeney, D. A., Anderson, K. L., Wuenschmann, A. and Jessen, C. R. 2017. Evaluation of canine hepatic masses by use of triphasic computed tomography and B-mode, color flow, power, and pulsed-wave Doppler ultrasonography and correlation with histopathologic classification. Am. J. Vet. Res. 78: 1273-1283. [Medline] [CrossRef]

6. Guillot, M., Danjou, M. A., Alexander, K., Bédard, C., Desnoyers, M., Beauregard, G. and Del Castillo, J. R. 2009. Can sonographic findings predict the results of liver aspirates in dogs with suspected liver disease? Vet. Radiol. Ultrasound 50: 513-518. [Medline] [CrossRef]

7. Jeon, T. Y., Kim, S. H., Lim, H. K. and Lee, W. J. 2010. Assessment of triple-phase CT findings for the differentiation of fat-deficient hepatic angiomyolipoma from hepatocellular carcinoma in non-cirrhotic liver. Eur. J. Radiol. 73: 601-606. [Medline] [CrossRef]

8. Ji, H., McTavish, J. D., Mortele, K. J., Wiesner, W. and Ros, P. R. 2001. Hepatic imaging with multidetector CT. Radiographics 21: S71-S80. [Medline] [CrossRef]

9. Jones, I. D., Lamb, C. R., Drees, R., Priestnall, S. L. and Mantis, P. 2016. Associations between dual-phase computed tomography features and histopathologic diagnoses in 52 dogs with hepatic or splenic masses. Vet. Radiol. Ultrasound 57: 144-153. [Medline] [CrossRef]

10. Kutara, K., Seki, M., Ishikawa, C., Sakai, M., Kagawa, Y., Iida, G., Ishigaki, K., Teshima, K., Edamura, K., Nakayama, T. and Asano, K. 2014. 
Triple-phase helical computed tomography in dogs with hepatic masses. Vet. Radiol. Ultrasound 55: 7-15. [Medline] [CrossRef]

11. Léveillé, R., Partington, B. P., Biller, D. S. and Miyabayashi, T. 1993. Complications after ultrasound-guided biopsy of abdominal structures in dogs and cats: 246 cases (1984-1991). J. Am. Vet. Med. Assoc. 203: 413-415. [Medline]

12. Miles, K. A. 1999. Tumour angiogenesis and its relation to contrast enhancement on computed tomography: a review. Eur. J. Radiol. 30: 198-205. [Medline] [CrossRef]

13. Murakami, T., Feeney, D. A. and Bahr, K. L. 2012. Analysis of clinical and ultrasonographic data by use of logistic regression models for prediction of malignant versus benign causes of ultrasonographically detected focal liver lesions in dogs. Am. J. Vet. Res. 73: 821-829. [Medline] [CrossRef]

14. Rothuizen, J. and Twedt, D. C. 2009. Liver biopsy techniques. Vet. Clin. North Am. Small Anim. Pract. 39: 469-480. [Medline] [CrossRef]

15. Sahani, D. V. and Singh, A. H. 2008. Dual-phase liver MDCT. pp. 83-92. In: MDCT from Protocol to Practice (Kalra, M. K., Saini, S. and Rubin G. D. eds.), Springer, Milan.

16. Scialpi, M., Volterrani, L., Mazzei, M. A., Cappabianca, S., Barberini, F., Piscioli, I., Brunese, L. and Lupattelli, L. 2009. Small $(<$ or $=2 \mathrm{~cm})$ atypical hepatic haemangiomas in the non-cirrhotic patient: pattern-based classification scheme for enhancement at triple-phase helical CT. Radiol. Med. (Torino) 114: 935-947. [Medline] [CrossRef]

17. Soyer, P., Poccard, M., Boudiaf, M., Abitbol, M., Hamzi, L., Panis, Y., Valleur, P. and Rymer, R. 2004. Detection of hypovascular hepatic metastases at triple-phase helical CT: sensitivity of phases and comparison with surgical and histopathologic findings. Radiology 231: 413-420. [Medline] [CrossRef]

18. Taniura, T., Marukawa, K., Yamada, K., Hikasa, Y. and Ito, K. 2009. Differential diagnosis of hepatic tumor-like lesions in dog by using dynamic CT scanning. Hiroshima J. Med. Sci. 58: 17-24. [Medline] 